\title{
The FREEDOM Study: does the Saga Continue for Diabetic Patients?
}

\author{
Marinella Centemero' ${ }^{1}$ Alexandre Abizaid ${ }^{2}$, J. Eduardo Sousa ${ }^{3}$
}

$\mathrm{T}$ he FREEDOM study, recently presented at American Heart Association (AHA) congress in November 2012 and simultaneously published in the prestigious New England Journal of Medicine, demonstrated that myocardial revascularisation surgery provides better results than percutaneous coronary intervention $(\mathrm{PCl})$ with drug-eluting stents (DES) for the treatment of diabetic patients with complex coronary artery disease. In this international multicenter study involving 140 hospitals, of which four are located in Brazil (Instituto Dante Pazzanese de Cardiologia - São Paulo, SP; Instituto do Coração do Hospital das Clínicas da Faculdade de Medicina da Universidade de São Paulo - São Paulo, SP; Hospital Universitário da Pontifícia Universidade Católica de Porto Alegre - Porto Alegre, RS; and Hospital Cardiológico Costantini - Curitiba, PR), 1,900 diabetic patients with multivessel involvement and no lesions in the left main coronary artery were randomized to coronary artery bypass graft (CABG) surgery or $\mathrm{PCl}$ procedures. ${ }^{1}$

At the end of the five-year follow-up (mean follow-up of 3.8 years), the primary study outcome, consisting of death from all causes, non-fatal myocardial infarction, and non-fatal stroke, occurred in $26.6 \%$ of the $\mathrm{PCl} /$ DES group vs. $18.7 \%$ in the CABG group ( $P=0.005)$, with a relative risk reduction of $30 \%$ in favor of CABG. Surgical revascularisation was also associated with lower rates of myocardial infarction $(6 \%$ vs. $13.9 \%$, $\mathrm{P}<0.001$ ), but with a significantly higher incidence of stroke $(5.2 \%$ vs. $2.4 \%$; $\mathrm{P}=0.03)$. There was also a marginal reduction of overall mortality in patients treated with CABG when compared to PCI/DES $(10.9 \%$ vs. $16.3 \% ; P=0.049)$.

Whether these results will have an impact on clinical practice and how they will influence future guidelines for the treatment of coronary heart disease in diabetic individuals remains to be determined.

The publication of this important study follows a series of previous studies regarding the question of the best available treatment for the management of patients with coronary artery disease and diabetes. This saga began in the 1990s with the first study, "Bypass Angioplasty Revascularization Investigation" (BARI), which warned the scientific community about the clinical and anatomical complexity of this subgroup and the great challenges that $\mathrm{PCl}$ (at that time characterized by the balloon angioplasty) represented for these patients. These challenges included incomplete revascularisation due to the difficulty of approaching certain types of lesions, such as total occlusions, and the high rates of coronary restenosis. ${ }^{2}$ These factors, together with the high cardiovascular risk inherent to diabetes patients, compromised the late clinical outcome and resulted in higher mortality in the percutaneous group. Interestingly, in patients with diabetes who were eligible for the study but were not randomized (BARI registry), for whom the best treatment strategy was decided by the physicians on an individual basis, there was no difference in mortality between the two types of revascularisation. ${ }^{3}$

Subsequently, during the nearly 20-year period following the published alert from National Heart, Lung, and Blood Institute (NHBLI) in September 1995, ${ }^{4}$ based on the results of BARI, several randomized trials, metaanalyses, and cohort studies analyzed the performance of diabetic patients treated by $\mathrm{CABG}$ or $\mathrm{PCl}$, with and without the use of DES, in the context of multivessel coronary disease. Among them, two recent randomized studies should be highlighted: Coronary Artery Revascularization in Diabetes (CARDia) and SYNergy Between PCI With TAXUS and Cardiac Surgery (SYNTAX). ${ }^{5,6}$

\footnotetext{
${ }^{1}$ Doctor. Clinical Cardiologist Physician at the Invasive Cardiology Service of Instituto Dante Pazzanese de Cardiologia. São Paulo, SP, Brasil.

2 Full Professor. Director of Invasive Cardiology Service of Instituto Dante Pazzanese de Cardiologia. São Paulo, SP, Brasil.

3 Full Professor. Director of Heart Structural Diseases Interventions Center of Instituto Dante Pazzanese de Cardiologia. São Paulo, SP, Brasil. Correspondence to: Marinella Centemero. Av. Dr. Dante Pazzanese,
}

500 - Vila Mariana - São Paulo, SP, Brasil - CEP 04012-909

E-mail: mpcentemero@yahoo.com.br

Received on: 1/12/2012 • Accepted on: 2/12/2012 
In the CARDia study, 510 diabetic patients with multivessel disease or complex single-vessel disease were randomized to receive $\mathrm{PCl}$ (sirolimus-DES were used in $70 \%$ of the patients) or CABG. After five years, the occurrences of the combined outcome of death, myocardial infarction, and stroke were similar in both groups $(26.6 \%$ vs. $20.5 \%$; $\mathrm{P}=0.11)$.

However, the rates of death, myocardial infarction, stroke, and the need for a second revascularization were significantly higher in the $\mathrm{PCl}$ group $(37.5 \%$ vs. $26 \%$, $P=0.005)$, leading the authors to conclude that the performance of percutaneous treatment in this subgroup was feasible and safe, although new interventions are necessary. ${ }^{5}$

In the SYNTAX study, which included 1,800 patients with complex coronary artery disease randomized to $\mathrm{CABG}$ or $\mathrm{PCI} / \mathrm{DES}$ (paclitaxel), 452 patients were diabetics $(25 \%)$, with higher-risk clinical profiles compared to non-diabetics (significantly higher percentage of women, arterial hypertension, dyslipidaemia, heart failure, peripheral vascular disease, and previous stroke). Nevertheless, the incidence of major events (death, myocardial infarction, and stroke) was similar in the CABG and $\mathrm{PCl}$ groups at the end of one-year ${ }^{6}(10.3 \%$ vs. $10.1 \%, P=0.96)$ and three-year $^{7}(16.3 \%$ vs. $14 \%$; $\mathrm{P}=0.53$ ) follow-ups. New procedures were significantly more frequent in diabetics treated with PCI/DES, both after one year $(20.3 \%$ vs. $6.4 \% ; \mathrm{P}<0.001)$ and three years of evolution (28\% vs. $12.9 \%$; $\mathrm{P}=0.001)$.

In summary, the CARDia and SYNTAX studies, which involved almost 1,000 diabetic patients, demonstrated that percutaneous treatment (in most cases with the use of DES) is as safe as CABG, since the combined rates of major adverse cardiac events were similar with the two techniques. Both studies also demonstrated that CABG surgery has the advantage of a decreased need for interventions in the short- and medium-term compared to $\mathrm{PCl}$. This fact is probably related to the greater anatomical complexity of the lesions treated in diabetic patients, which consequently result in higher rates of restenosis and incomplete revascularization, even with the use of DES. The accelerated progression of coronary atherosclerosis is also a contributing factor to the need for repeat revascularizations in this subgroup.

Upon detailed analysis of the FREEDOM study, several virtues can be identified:

1. It was the first randomized, multicenter study specifically designed to evaluate both types of revascularization (percutaneous vs. surgical) exclusively in diabetic patients with complex coronary artery disease.

2. Both $\mathrm{CABG}$ and $\mathrm{PCl}$ were performed according to contemporary practice available at the time (20052010).

3. It was a superiority trial, which provides great statistical value.
4. It was sponsored by an independent institution of unquestionable prestige (NHLBI).

5. To date, the study has involved the largest number of patients with a confirmed diabetes diagnosis $(1,900)$, randomized for the comparison of the two types of revascularisation.

6. The majority of patients (approximately two-thirds) had triple vessel disease.

7. There was great concern regarding the control of risk factors related to coronary artery disease, particularly diabetes, emphasizing the achievement of stringent goals established by the international guidelines of cardiology and the American Diabetes Association (ADA).

However, as for all studies, even those carefully designed to fit in the canons of evidence-based medicine, critical analysis can and should be performed. Therefore, the following considerations regarding the FREEDOM trial aim to exercise clinical reasoning, drawing upon the experience gained during the many years of daily practice since the beginning of interventional cardiology, as well as active participation in several randomized studies.

It is noteworthy that approximately 33,000 diabetic patients were screened to participate in the survey, but only $10 \%(3,309)$ were eligible, of whom 1,900 $(57 \%)$ agreed to fully participate in the study. Thus, their conclusions refer specifically to this population. There is no information regarding the treatment given to the diabetics excluded from the study or to those who refused randomization, although eligible. A "FREEDOM registry" showing the revascularization technique employed and the evolution of these patients, similar to what the SYNTAX and BARI studies showed, would be desirable and enlightening.

Throughout the five-year recruitment period, the study was amended twice - in 2007 and 2009 - with regard to sample size, duration of clinical follow-up, and the rate of major cardiovascular events, probably due to the long randomization time (2005-2010) and the low rate of patient recruitment. Initially, 2,400 diabetic patients were predicted to be enrolled during a two-year period, with a minimum follow-up of three years, to ensure $85 \%$ power to detect a relative reduction in the primary endpoint of $18 \%$ to $23 \%$ after four years. The study ended with 1,900 patients who were followed for 4.75 years, with a minimum follow-up of two years and an estimated power of $80 \%$ to detect a $27 \%$ relative reduction in event rates for the two treatment groups.

Regarding the results, it is noteworthy that the mortality of diabetic patients undergoing CABG surgery at 30 days in the FREEDOM trial was only $1.7 \%$, an extremely low percentage that is most likely difficult to reproduce in daily practice outside of the ideal conditions of a randomized study. 
Accordingly, we cite the Brazilian experience, well documented by Braile and Gomes, ${ }^{8}$ which reveals that the average mortality for Coronary artery bypass graft (CABG) surgery in Brazil, based on data provided by the Department of Informatics of the Brazilian Unified Health System (Banco de Dados do Sistema Único de Saúde - DATASUS), is approximately 4.8\%. In Europe, these rates vary from $3 \%$ to $7.3 \%$.

In another article, published in 2009, that collected data on the Brazilian experience on CABG from the database of the Hospital Information System of the Sistema de Informações Hospitalares do Sistema Único de Saúde (SIH/SUS) from 2005 to 2007, Piegas et al. ${ }^{9}$ reported that 63,529 procedures were performed in 191 hospitals. The overall in-hospital mortality was $6.2 \%$, and it was higher in hospitals with lower surgical volume when compared to that observed in larger-volume hospitals $(7.29 \%$ vs. $5.77 \%, \mathrm{P}<0.001)$. Comparatively, these authors cited data from the American registry "Adult Cardiac Surgery in New York State" 2003-2005, which observed a 30 -day mortality rate of $2.14 \%$. The authors also cited the mortality rates of the member countries of the project "European System for Cardiac design Operative Risk Evaluation (EuroSCORE)." These rates were as follows: Germany, 2.4\%; United Kingdom, $3.7 \%$; Spain, 6.8\%; Italy, 2.4\%; France, 3.2\%; and Finland, $1.5 \%$ (mean of $3.4 \%){ }^{9}$

Another interesting finding concerns the analysis of the combined primary endpoint of the study according to several pre-specified subgroups. Outside of the United States, the occurrence of major cardiac events at five years was similar for PCI/DES and CABG (25\% vs. $21 \%, P=N S$ ). The explanation for this regional difference remains unclear.

After such considerations, it appears that there is no consensus regarding the best option for multivessel revascularization in diabetic patients. Revascularization strategies should be individualized according to other variables, such as patient fragility, risk of stroke, presence of comorbidities (such as kidney disease and chronic obstructive pulmonary disease), experience of the interventional and surgical teams, patient socioeconomic status, and adherence to pharmacological treatment, which may influence the early and late results of the chosen revascularization.
In conclusion, we would like to emphasize that the surgical and percutaneous procedures are complementary during the course of coronary artery disease. Discussions between clinicians, surgeons, and interventional (the so-called "heart team") are highly recommended to assess the advantages and disadvantages of both types of revascularization. Additionally, the patient and his/ her family should also be involved in the decisionmaking process, and should be honestly informed about the available revascularization options and their risks.

\section{CONFLICT OF INTEREST}

The authors declare no conflicts of interest.

\section{REFERENCES}

1. Farkouh ME, Domanski M, Sleeper LA, Siami FS, Dangas G, Mack M, et al.; FREEDOM Trial Investigators. Strategies for multivessel revascularization in patients with diabetes. N Engl J Med. 2012;367(25):2375-84

2. Comparison of coronary bypass surgery with angioplasty in patients with multivessel disease. The Bypass Angioplasty Revascularization Investigation (BARI) Investigators. N Engl Med. 1996;335(4):217-25

3. Feit F, Brooks MM, Sopko G, Keller NM, Rosen A, Krone R, et al. Long-term clinical outcome in the Bypass Angioplasty Revascularization Investigation Registry: comparison with the randomized trial. BARI Investigators. Circulation. 2000;101(24):2795-802.

4. Clinical alert: bypass over angioplasty for patients with diabetes. Del Med J. 1995;67(11):594.

5. Hall R, Serruys P. CARDia: Coronary Artery Revascularization in Diabetes trial. In: ESC Congress 2012. [cited 11 Jan 2012]. Available from: http://www.escardio.org/congresses/esc-2012/ congress-reports/Pages/710-5-CARDia.aspx

6. Banning AP, Westaby S, Morice MC, Kappetein AP, Mohr FW, Berti $S$, et al. Diabetic and nondiabetic patients with left main and/or 3-vessel coronary artery disease: comparison of outcomes with cardiac surgery and paclitaxel-eluting stents. J Am Coll Cardiol. 2010;55(11):1067-75.

7. Kappetein AP, Feldman TE, Mack MJ, Morice MC, Holmes DR, Ståhle $\mathrm{E}$, et al. Comparison of coronary bypass surgery with drug-eluting stenting for the treatment of left main and/or three-vessel disease: 3-year follow-up of the SYNTAX trial. Eur Heart J. 2011;32(17):2125-34.

8. Braile DM, Gomes WJ. Evolução da cirurgia cardiovascular: a saga brasileira. Uma história de trabalho, pioneirismo e sucesso [editorial]. Arq Bras Cardiol. 2010;94(2):151-2.

9. Piegas LS, Bittar OJNV, Haddad N. Cirurgia de revascularização miocárdica: resultados do Sistema Único de Saúde. Arq Bras Cardiol. 2009;93(5):555-60. 